\title{
STERILIZATION PROTOCOL FOR POROUS DENTAL IMPLANTS MADE BY SELECTIVE LASER MELTING
}

\author{
AVRAM MANEA ${ }^{1}$, SIMION BRAN ${ }^{2}$, MIHAELA BACIUT ${ }^{2}$, \\ GABRIEL ARMENCEA ${ }^{1}$, DUMITRU POP ${ }^{3}$, PETRU BERCE ${ }^{4}$, \\ DAN-CRISTIAN VODNAR ${ }^{5}$, MIHAELA HEDESIU ${ }^{1}$, CRISTIAN DINU ${ }^{1}$, \\ ADRIAN PETRUTIU ${ }^{2}$, DARIUS TOMINA ${ }^{2}$, GRIGORE BACIUT ${ }^{1}$
}

\author{
${ }^{1}$ Department I - Maxillo-Facial Surgery and Radiology, Iuliu Hatieganu University \\ of Medicine and Pharmacy, Cluj-Napoca, Romania \\ ${ }^{2}$ Department III - Oral Rehabilitation, Iuliu Hatieganu University of Medicine and \\ Pharmacy, Cluj-Napoca, Romania \\ ${ }^{3}$ Department of Mechanical Systems Engineering, Technical University of Cluj- \\ Napoca, Romania \\ ${ }^{4}$ Department of Engineering Fabrication, Technical University of Cluj-Napoca, \\ Romania \\ ${ }^{5}$ Faculty of Food Science and Technology, University of Agricultural Sciences and \\ Veterinary Medicine of Cluj-Napoca, Romania
}

\section{Abstract}

Background and aims. Although dental implants are widely and successfully used, failure rates because of bacterial colonization are still high. Adequate fabrication and sterilization techniques as well as proper management of infectious complications represent a constant field of interest and research. In this study, we focused our attention on implants with controlled porosity produced by Selective Laser Melting (SLM). The difficulty to sterilize porous implantable devices is well known and finding an adequate sterilization protocol represents a challenge worldwide. Before testing the biological and mechanical properties of porous implants, a preliminary study in order to determine a correct sterilization protocol must be conducted.

Our aim was to establish a valid sterilization protocol for porous titanium alloy dental implants, as such protocols are not officially available yet.

Methods. Twenty dental implants were fabricated from a titanium alloy by SLM. Ten of them were made using a $150 \mathrm{~W}$ laser beam (porosity of $1 \%$ - group A) and the rest using a $75 \mathrm{~W}$ laser beam (porosity of $23 \%$ - Group B), all of them with a non-defined internal structure. The implants were initially sterilized (5 from group A and 5 from group $B$, using dry heat $-180^{\circ} \mathrm{C}$ for 2 hours; the rest using steam sterilization $-121^{\circ} \mathrm{C}$ for $15 \mathrm{~min}$ ) and then spent 18 hours in culture media with developing bacteria (Bacillus cereus (ATCC 11778), Staphylococcus aureus (ATCC 49444), Enterococcus faecalis (ATCC 29212), Listeria monocytogenes (ATCC 19114), three Gram negative bacteria: Escherichia coli (ATCC 25922), Salmonella typhimurium (ATCC 14028) and Pseudomonas aeruginosa (ATCC 27853). The first ten implants (5 from group A and 5 from group B) were then sterilized with dry heat and the others with steam. After sterilization, they were all placed in sterile culture media in order to observe if any bacterial growth were present.

Results. The culture media was observed 18 hours after the sterilized implants were placed inside. No bacterial growth was observed.

Conclusions. Our tests reached their aims of defining a protocol to sterilize porous implants. Future tests regarding biological and mechanical aspects of these implants may now follow.

Keywords: selective laser melting, dental implants, porous implants, sterilization

Manuscript received: 14.02 .2018

Received in revised form: 29.06.2018

Accepted: 10.07.2018

Address for correspondence: avram.manea@umfcluj.ro 


\section{Background and aims}

Efficient sterilization techniques have been available for decades, and their impact in the development of medical implants has been of crucial importance. According to ISO 17665-1:2006, a sterile medical device is one which is free of viable microorganisms. The same standard states that: "The purpose of sterilization is to inactivate the microbiological contaminants and thereby transform the non-sterile products into sterile ones." Before being able to place any sort of implant in the human body, proper sterilization must be obtained. In the past, implants were not considered a viable solution due to their high failure rate. Researchers then realized that it was not the implant itself that was causing the complications, but rather the bacteria on its surface. Once viable sterilization protocols were established, medical and especially oral implantology became more and more popular with both practitioners and patients [1,2].

Porous implants made using Selective Laser Melting (SLM) present numerous advantages $[3,4]$. This porosity is not limited only to the surface of the implant. Since it is made using an additive technique, the whole body of the implant has the same porosity. First of all, they are lighter. While this aspect is not as relevant for small implants (e.g. dental implants), the difference in weight is considerable in larger implants (used to reconstruct different anatomical regions - e.g. zygomatic complex defects). Also, since they are designed by computer software that reads the patient Cone Beam Computer Tomography (CBCT), the implants will fit perfectly into the defects, with no intraoperative adjustments required. This also reduces the operative time and provides the premises of improved clinical outcome [5]. The contact surface between implant and bone is increased with porous implants in comparison with regular, smooth surfaced implants. Bone cells will also develop inside the implant, into the porous network, not only at the surface [6-10]. In the case of dental implants, this means a larger area to spread the forces applied onto it, meaning less bone resorption due to high mechanical stress. Porous implants have a decreased elastic modulus which avoids the stress shield effect when fixated in the bone [9]. Last but not least, porous implants can serve as a reservoir for several drugs such as antibiotics. If the drug coating is only placed on the surface of the implant, its efficiency will be of short term. If the drugs are inside the pores of the implant, the release rate will be lower, more controllable and the effect will last much longer [10-12]. In the case of dental implants, such substances can lower the risks of peri-implantitis which is one of the leading causes for implant loss [13-15].

Studies show the importance of systemic drugs in the process of osseointegration of different types of titanium implants. They can either impair or enhance the process. Knowing their effect can increase implant survival rates and reduce complication rates. Anabolic and anticatabolic bone-acting agents proved to have a positive effect, while nonsteroidal anti-inflammatory drugs, proton pump inhibitors and selective serotonin reuptake inhibitors, frequently administered after surgery, might impair osseointegration. The effect of other frequently used drugs like aspirin, opioids, anticoagulants and antibiotics in implant fixations are yet not fully understood but worth studying [16]. These substances proved to influence the process of osseointegration when administrated systemically, but might not be as efficient when used as coatings for classical implants, due to their short term effect. When filling porous implants, the available quantity of drugs increases and the releasing time is also extended.

The use of SLM in manufacturing dental implants is a relatively new idea. Most of them are made of pure titanium and especially titanium alloys. SLM represents an additive technique that provides numerous advantages when it comes to dental implants (definable porosity gradient, roughness, customized geometry that cannot be obtained through classical subtractive methods). Porous implants produced by SLM have a stiffness (evaluated by Young's modulus) that is much lower than the bulk material. For instance, in the case of Ti-6Al-4V, the value of Young's modulus for the bulk material is $115 \mathrm{GPa}$ and for pure titanium $112 \mathrm{GPa}$. The bone's Young's modulus range is between 10 and $26 \mathrm{GPa}$. This difference causes the phenomenon of stress shielding, with a negative impact on the dental implant longevity. A $29 \%$ porous dental implant has a Young's modulus of 46 $\mathrm{GPa}$, which is much closer to the bone [15]. The internal structure of this type of implant can be defined by the user or it cannot be defined and so it results from the different powers of the laser beam. For instance a $100 \mathrm{~W}$ power will result in an actual porosity of $12.74 \%$ (14.7\% for the simulated model), while a $200 \mathrm{~W}$ laser power provides a $0.15 \%$ porosity $(0.4 \%$ for the simulated model) $[17,18,19]$.

Positively charged implant surfaces make them hydrophilic. This promotes the adherence of proteins at the bone-implant interface and stimulates osteoblasts function. These processes are important mainly in the early stages of osseointegration. Factors that affect surface energy are roughness, surface treatment, implant composition, sterilization, and handling during implantation. Porous implants produced by SLM present different such parameters compared to implants produced by standard methods (additive or subtractive). Further studies regarding the surface of porous implants are currently in progress, in order to evaluate these factors [20].

There is a strong current trend to produce standardized porous implants on a large scale. They can be sterilized using irradiation (Gamma and E-beam), plasma and chemicals (peracetic acid) and many other modalities [21,22]. All these procedures are efficient but involve great costs and are not available everywhere. While they prove to be the best solution for standardized implants, this cannot be said about custom made implants (dental or for other purpose). The fact that porous implants can be sterilized in an autoclave which is available in every clinic or dental practice means that, 


\section{Dental Medicine}

although sterilization is a vital step before their insertion, it is not a major inconvenience. It can be done in a short amount of time with minimal costs. Heat sterilization proved to be a good approach for porous structures (e.g. biodegradable scaffolds) in other studies as well. Still, it is important to take into account the amount of time expected to pass from the moment of sterilization to the time of implantation because moist heat sterilization for packed objects is only guaranteed for one month (versus two years for radiation sterilization), according to ISO standards [23].

Although the advantages of porous implants are obvious, some questions must still be answered: can they be properly sterilized since they present a cavernous $3 \mathrm{D}$ structure that might provide shelter for bacteria and what sterilization protocol is best fitted? Can they be sterilized on a smaller scale (each customized implant by itself) or do they require more complex sterilization techniques?

Adequate fabrication and sterilization techniques as well as proper management of infectious complications represent a constant field of interest and research. In this study, we focused our attention on implants with controlled porosity produced by Selective Laser Melting (SLM). The difficulty to sterilize porous implantable devices is well known and finding an adequate sterilization protocol represents a challenge worldwide. Before testing the biological and mechanical properties of porous implants, a preliminary study in order to determine a correct sterilization protocol must be conducted.

Our aim was to establish a valid sterilization protocol for porous titanium alloy dental implants as such protocols are not officially available yet.

\section{Methods}

Twenty dental implants were made from Ti6Al7Nb powder (a frequently used titanium alloy for implantable medical devices i.e. dental implants, hip prostheses etc. [9]) by Selective Laser Melting at the Technical University of Cluj-Napoca using the 250 SLM ReaLizer (ReaLizer $\mathrm{GmbH}$, Germany). Ten of them were made using a $150 \mathrm{~W}$ laser beam. This resulted in a $1 \%$ theoretical porosity - group A. The others were manufactured using a $75 \mathrm{~W}$ laser beam which resulted in a theoretical porosity of $23 \%$ - Group B. The actual porosity of the implants was not measured for this study, since it would not influence the results. All of them had a non-defined internal structure.

The implants were divided into two groups: I and II. Group I contained 5 implants from group A (1\% porosity) and 5 from group B ( $23 \%$ porosity). So did group II. Group I underwent an initial dry heat sterilization $\left(180^{\circ} \mathrm{C}\right.$, 2 hours) and group II was subject to steam sterilization in an autoclave $\left(121^{\circ} \mathrm{C}, 15 \mathrm{~min}\right)$. The sterile implants from the two groups were placed in culture media designed for this study. Ten strains were used: Bacillus cereus (ATCC 11778), Staphylococcus aureus (ATCC 49444), Enterococcus faecalis (ATCC 29212), Listeria monocytogenes (ATCC
19114), three Gram negative bacteria: Escherichia coli (ATCC 25922), Salmonella typhimurium (ATCC 14028) and Pseudomonas aeruginosa (ATCC 27853). All cultures were placed on Mueller Hinton agar (BioMérieux, Marcy l'Etoile, France). The bacteria were cultured overnight in $5 \mathrm{ml}$ Mueller Hinton broth (BioMérieux, Marcy l'Etoile, France) in a shaker incubator (HeidolphInkubator 1000 coupled with HeidolphUnimax 1010 , Schawbach, Germany) at $37^{\circ} \mathrm{C}, 150$ rpm until the culture was formed. An inoculation loop was first sterilized by passing it through a flame. When the loop was cool, it was dipped into the Mueller Hinton broth. The inoculation loop was then dragged across the surface of Petri dishes containing the agar (Muller Hinton agar) using the three-phase streaking pattern, known as the T-Streak. The technique is used to isolate a pure strain from a single species of microorganism. The dishes were incubated at $37^{\circ} \mathrm{C}$ for $18 \mathrm{~h}$. A sufficient number of colonies were removed and placed into $9 \mathrm{ml}$ of sterile saline. The bacterial concentration corresponding to $107 \mathrm{CFU}$ (Colony Forming Unit) /ml was established using the Nanodrop Spectrophotometer ND1000 , Delaware, USA. Successive dilutions up to 105CFU/ $\mathrm{ml}$ were obtained.

The bacterial adherence was tested to simulate the affinity of microorganisms to the specimen surface. Each bacteria was precultured from a frozen glycerol preparation and inoculated in $45 \mathrm{~mL}$ Tryptic Soy Broth (Liofilchem, RosetoDegli Abruzzi, Italy) of $16 \mathrm{~h}$ at $37^{\circ} \mathrm{C}$. Then, the sterilized specimens were placed in $15 \mathrm{ml}$ test tubes with $9 \mathrm{ml}$ Tryptic Soy Broth, $1 \mathrm{~mL}$ of bacterial suspension and the specimens. After incubation (HeidolphUnimax 1010, Schwabach, Germany) at $37^{\circ} \mathrm{C}$ for 18 hours, the specimens were removed and sterilized $\left(121^{\circ} \mathrm{C} .15 \mathrm{~min}\right.$ and $180^{\circ} \mathrm{C}, 2$ hours respectively). After sterilization the specimens were placed into $9 \mathrm{~mL}$ of Tryptic Soy Broth and incubated for 18 $\mathrm{h}$ at $37^{\circ} \mathrm{C}$. After incubation $0,1 \mathrm{~mL}$ from media were placed into Muller Hinton Agar plate and incubated for $18 \mathrm{~h}$ at $37^{\circ} \mathrm{C}$. The results are expressed as $\mathrm{CFU} / \mathrm{mL}$.

\section{Results}

One of the specimens developed several bacterial strains but the test was repeated for that certain implant and the results were negative. This might be due to external contamination during the manipulation of that specific specimen. All the other culture media showed negative results as can be seen in Table I.

Table I. Concentration of bacteria found on the implants after sterilization.

\begin{tabular}{|l|l|}
\hline Strain & CFU/ML \\
\hline Staphylococcus aureus (ATCC 49444), & 0 \\
\hline Bacillus cereus (ATCC 11778), & 0 \\
\hline Listeria monocytogenes (ATCC 19114), & 0 \\
\hline Enterococcus faecalis (ATCC 29212), & 0 \\
\hline Pseudomonas aeruginosa (ATCC 27853), & 0 \\
\hline Salmonella typhimurium (ATCC 14028) & 0 \\
\hline Escherichia coli (ATCC 25922) & 0 \\
\hline
\end{tabular}


No additional sterilization was required. Dry heat and steam sterilization both proved to be efficient in the case of porous dental implants produced by SLM.

\section{Discussion}

Although dental implants are widely and successfully used, failure rates resulting from bacterial colonization are still high [23]. Adequate fabrication and sterilization techniques as well as proper management of infectious complications represent a constant field of interest and research.

Recent studies concerning dental implant sterilization propose different protocols, like hydrothermal sterilization, in order to avoid certain disadvantages of current techniques [24]. Other studies follow the effects of most sterilization techniques on the implant's properties [22]. Standard sterilization protocols are available for all the current techniques: ISO 17665-1:2006 and ISO 17665-2:2006 for moist heat; ISO 20857:2010 for dry heat; ISO 11135:2014 for ethylene oxide; ISO 11137-2:2013 for radiation etc. Still, no clear protocol for porous implant sterilization is currently available.

Implants with controlled porosity produced by Selective Laser Melting (SLM) are currently subject of research for better mechanical and biological properties. The difficulty to sterilize porous implantable devices is well known and finding an adequate sterilization protocol represents a challenge worldwide [25].

The issue of implant sterilization is studied in several other fields, like orthopedics. For many decades, implants were sterilized using the autoclave, taking it for granted, but researchers decided that the efficiency of using standard sterilization protocols in certain situations needs to be tested [24].

Important questions are also raised concerning the origin and pathway of colonization or infection of the implantable devices, pre-, intra- or postoperatively. The role of the superficial microbial biofilm rather than internal structural colonization was pointed out in several studies $[24,26,27]$.

As such, before testing the biological and mechanical properties of porous implants, a preliminary study in order to determine a correct sterilization protocol must be conducted.

Our aim was to establish a valid sterilization protocol for porous titanium alloy implants, as such protocols are not officially available yet.

We conducted dry heat and moist heat sterilization tests, as recommended by the conclusions of the Govindaraj and Muthuraman [22] and Kilpadi et al. [28] study groups. They showed that dry heat has several advantages like: increased penetration power, noncorrosive for metal objects, positive effects on titanium objects' properties, nontoxic and eco-friendly and also cost efficient. Moist heat (or autoclaving) was the first standardized sterilization method (ISO 11134:1994). It is a fast, efficient, simple and reliable technique $[22,28]$.

Considerable research was conducted on the issue of variation in sterilization capacity on the surface and the interior of implants [22,24,25,28].

No bacteria remained active on the surface or inside the porous implants in our study after conventional sterilization (dry and moist heat).

Sterilization in oral implantology has been considered efficient for decades, but recent studies revisited this issue, in order to establish better protocols or to obtain improved implant characteristics [24].

Dry and moist heat sterilization are known to have good penetration rates, higher than other, more expensive methods. Although short steam cycles are not recommended by other studies for implantable objects [28], our study shows that they can be as efficient as other more time consuming methods, from the point of view of the bacterial strains that were tested. Also, porous structures are mentioned as being at risk of not obtaining an efficient sterilization in most types of autoclaves but results can be improved by adding a vacuum pump to increase penetration [29]. This theory is confirmed in our present study by the fact that a complete sterilization was obtained, even with a faster cycle, by using a vacuum autoclave. Gibraltar Laboratories recommend a sterilization temperature for porous items of $132^{\circ} \mathrm{C}$ or $135^{\circ} \mathrm{C}$ [30]. Our findings indicate that an even lower temperature $\left(121^{\circ} \mathrm{C}\right)$ can be enough.

Our results are similar to those of other studies following the same issue [24]. Wiliams et al. used a similar testing protocol. They used porous coated and smooth titanium cut into diamond shaped cupons (opposed to the implants we used, that presented a porous structure in their entire body, not only at the surface), and autoclaving temperatures of $121^{\circ} \mathrm{C}$ and $132^{\circ} \mathrm{C}$, in short $(5-10$ minutes) cycles. The number of bacterial strains was lower than in our study (Staphylococcus aureus (MRSA) and Bacillus subtilis), and also more specific to orthopedic infections rather than the bacteria more commonly found in the oral cavity. As in our protocol, the objects that underwent sterilization were placed in sterile culture media and bacterial growth was observed. The $121{ }^{\circ} \mathrm{C}$ short (5 minutes) protocols were proved to be inefficient, as MRSA cells were found to survive on both porous and smooth surfaces. Their recommendation for sterilization of implants that have had associated infection and are considered for reimplantation is the use of moist heat at $132^{\circ} \mathrm{C}$ for longer than 10 minutes. Another important aspect of this study is the fact that, although higher temperatures and increased duration will make the biofilms non-viable, their presence cannot be removed by any possible autoclave setting [24].

There is a strong current trend to produce standardized porous implants on a large scale. They can be sterilized using irradiation (Gamma and E-beam), plasma and chemicals (peracetic acid) and many other modalities 
$[21,22]$. All these procedures are efficient but involve great costs and are not available everywhere. While they prove to be the best solution for standardized implants, this cannot be said about custom made implants (dental or for other purpose). The fact that porous implants can be sterilized in an autoclave which is available in every clinic or dental practice means that, although sterilization is a vital step before their insertion, it is not a major inconvenience. It can be done in a short amount of time with minimal costs. Heat sterilization proved to be a good approach for porous structures (e.g. biodegradable scaffolds) in other studies as well. Still, it is important to take into account the amount of time expected to pass from the moment of sterilization to the time of implantation because moist heat sterilization for packed objects is only guaranteed for one month (versus two years for radiation sterilization), according to ISO standards [24].

Our study shows that the methods used for sterilizing classical dental implants and even dental instruments can be applied in the case of porous implants, which makes their usage very convenient [31].

\section{Conclusion}

Our preliminary study shows that porous implants (namely dental implants) made from titanium alloy by SLM can be adequately sterilized using common and affordable sterilization techniques (moist heat, dry heat), which are available in any clinic or dental practice. The present experiment consolidates the fact that adequate surface and intrinsic sterilization can be ensured with these conventional sterilization methods.

An easy sterilization guide for porous implants can be formulated this way.

Avoidance of costly and complicated sterilization procedures (irradiation, chemical protocols) is of great advantage as proper preparation allows for higher clinical survival and success rates. Future research in this field is now enabled, once experimental and clinical use can be performed safely.

\section{References}

1. Levin L. Dealing with dental implant failures. J Appl Oral Sci. 2008;16(3):171-175.

2. Elian N. Dental Implants: Past, Present, and Future. Compendium of Continuing Education in Dentistry. 2009 Sep; 30(7): 388-408.

3. Li Y, Yang C, Zhao H, Qu S, Li X, Li Y. New Developments of Ti-Based Alloys for Biomedical Applications. Materials (Basel). 2014;7(3):1709-1800.

4. Armencea G, Berce C, Rotaru H, Bran S, Leordean D, Coada $\mathrm{C}$, et al. Micro-CT and histological analysis of Ti6A17Nb custom made implants with hydroxyapatite and $\mathrm{SiO} 2-\mathrm{TiO} 2$ coatings in a rabbit model.Clujul Med. 2015;88(3):408-414.

5. Rotaru H, Schumacher R, Kim SG, Dinu C. Selective laser melted titanium implants: a new technique for the reconstruction of extensive zygomatic complex defects. Maxillofac Plast Reconstr Surg. 2015 Jan 29;37(1):1. doi: 10. 1186/ s40902-015-
0001-9.

6. Blinova MI, Yudintzeva NM, Nikolaenko NS, Potokin IL, Raykhtsaum G, Pitkin MR, et al. Cell Cultivation on Porous Titanium Implants with Various Structures. Cell Tissue Biol. 2010;4(6):572-579.

7. Shibata Y, Tanimoto Y, Maruyama N, Nagakura M. A review of improved fixation methods for dental implants. Part II: biomechanical integrity at bone-implant interface. J Prosthodont Res. 2015;59(2):84-95.

8. Lucaciu O, Soriţău O, Gheban D, Ciuca DR, Virtic O, Vulpoi A, et al. Dental follicle stem cells in bone regeneration on titanium implants. BMC Biotechnol. 2015 Dec 30;15:114. doi: 10.1186/ s12896-015-0229-6.

9. Armencea G, Berce C, Rotaru H, Bran S, Leordean D, et al. Titanium alloys with hydroxyapatite or $\mathrm{SiO} 2+\mathrm{TiO} 2$ coatings used in bone reconstruction. Optoelectron Adv Mat. 2015;9(5):865868.

10. Shi M, Kretlow JD, Spicer PP, Tabata Y, Demian N, Wong $\mathrm{ME}$, et al. Antibiotic-releasing porous polymethylmethacrylate/ gelatin/antibiotic constructs for craniofacial tissue engineering. J Control Release. 2011;152(1):196-205.

11. Lazar MA, Vodnar D, Prodan D, Rotaru H, Roman CR, Sorcoi LA, et al. Antibacterial coating on biocomposites for cranio-facial reconstruction. Clujul Med. 2016;89(3):430-434.

12. Shi M, Kretlow JD, Nguyen A, Young S, Scott Baggett L, Wong $\mathrm{ME}$, et al. Antibiotic-releasing porous polymethylmethacrylate constructs for osseous space maintenance and infection control. Biomaterials. 2010;31(14):4146-4156.

13. Alhilou A, Do T, Mizban L, Clarkson BH, Wood DJ, Katsikogianni MG. Physicochemical and Antibacterial Characterization of a Novel Fluorapatite Coating. ACS Omega. 2016;1(2):264-276.

14. He S, Zhou P, Wang L, Xiong X, Zhang Y, Deng Y, et al. Antibiotic-decorated titanium with enhanced antibacterial activity through adhesive polydopamine for dental/bone implant. J R Soc Interface. 2014 Mar 19; 11(95): 20140169. doi: 10.1098/ rsif.2014.0169.

15. Mahato N, Wu X, Wang L. Management of peri-implantitis: a systematic review, 2010-2015. Springerplus. 2016 Feb 1;5:105. doi: 10.1186/s40064-016-1735-2.

16. Apostu D, Lucaciu O, Lucaciu GD, Crisan B, Crisan L, Baciut M, et al. Systemic drugs that influence titanium implant osseointegration. Drug Metab Rev. 2017;49(1):92-104.

17. Cardaropoli F, Alfieri V, Fabrizia C, Vincenzo S. Manufacturing of Porous Biomaterials for Dental Implant Applications through Selective Laser Melting. Advanced Materials Research. June 2012; 535-537.

18. Laakso P, Riipinen T, Laukkanen A, Andersson T, Jokinen A, Revuelta A, Ruusuvuori K. Optimization and Simulation of SLM Process for High Density H13 Tool Steel Parts. Physics Procedia. Dec 2016. 83:26-35.

19. Hanzl P, Zetek M, Bakša T, Kroupa T. The Influence of Processing Parameters on the Mechanical Properties of SLM Parts. Dec 2015 Procedia Engineering 100.

20. Apostu D, Lucaciu O, Berce C, Lucaciu D, Cosma D. Current methods of preventing aseptic loosening and improving osseointegration of titanium implants in cementless total hip arthroplasty: a review. J Int Med Res. 2017 Jan 1:300060517732697. doi:10.1177/0300060517732697.[Epub ahead of print].

21. Dai Z, Ronholm J, Tian Y, Sethi B, Cao X. Sterilization techniques for biodegradable scaffolds in tissue engineering 
applications. J Tissue Eng. 2016 May 17;7:2041731416648810. doi: $10.1177 / 2041731416648810$.

22. Govindaraj S, Muthuraman MS. Systematic Review on Sterilization Methods of Implants and Medical Devices. International Journal of Chem Tech Research. 2015;8:897-911.

23. Rakic M, Galindo-Moreno P, Monje A, Radovanovic S, Wang HL, Cochran D, et al. How frequent does peri-implantitis occur? A systematic review and meta-analysis. Clin Oral Investig. 2018;22(4):1805-1816.

24. Shi X, Xu L, Wang Q, Sunarso, Xu L. Hydrothermal Sterilization Improves Initial Osteoblast Responses on SandpaperPolished Titanium. Materials (Basel). 2017 Jul 17;10(7). pii: E812. doi: 10.3390/ma10070812.

25. Williams DL, Taylor NB, Epperson RT, Rothberg DL. Flash autoclave settings may influence eradication but not presence of well-established biofilms on orthopaedic implant material. J Orthop Res. 2017 Oct 4. doi: 10.1002/jor.23764. [Epub ahead of print]

26. Stacchi C, Berton F, Porrelli D, Lombardi T. Reuse of Implant Healing Abutments: Comparative Evaluation of the Efficacy of Two Cleaning Procedures. Int J Prosthodont. 2018;31(2):161-162.
27. Canullo L, Tallarico M, Chu S, Peñarrocha D, Özcan M, Pesce P. Cleaning, Disinfection, and Sterilization Protocols Employed for Customized Implant Abutments: An International Survey of 100 Universities Worldwide. Int J Oral Maxillofac Implants. 2017;32(4):774-778.

28. Kilpadi D V,Weimer J J, Lemons J E. Effect of passivation and dry heat sterilization on surface energy and topography of unalloyed titanium implants, Colloids and surfaces A: Physicochemical and Engineering Aspects 1998; 135: 89-101. 29. Rutala WA, Weber DJ. Healthcare Infection Control Practices Advisory Committee (HICPAC). Guideline for Disinfection and Sterilization in Healthcare Facilities 2008; 8-9.

30. Mastej J. Instrument Sterilization and Cleaning. Reprocessing Reusable Medical Devices. Cleaning and Moist Heat Steam Sterilization Validation Process. Gibraltar Laboratories. 27 Feb. 2018 Available at: https://www.omtecexpo.com/images/stories/ OMTEC-2015_Mastej_Sterilization.pdf

31. Infection Control. Centers for Disease Control and Prevention, Centers for Disease Control and Prevention, 27 Feb. 2018, available at: www.cdc.gov/infectioncontrol/guidelines/ disinfection/sterilization/steam.html. 